\title{
Atenuación con disminución prosódica significativa en géneros con distinto grado de planificación discursiva
}

\author{
Adrian Cabedo \\ Universitat de València
}

\begin{abstract}
Este estudio analiza la frecuencia de grupos entonativos con disminución prosódica y atenuación pragmática en tres géneros discursivos del español: la conversación coloquial, la entrevista televisiva y el monólogo humorístico. En total, se han recogido 3365 grupos entonativos, de los que 237 han manifestado valores reducidos fónicamente; el criterio de discriminación ha sido que los valores de velocidad elocutiva, rango tonal o intensidad se distancien 1.65 desviaciones típicas de la media del hablante. Los datos indican que entre un 15 y un $20 \%$ de grupos entonativos por género utilizan disminuciones prosódicas y que, de ellos, la mitad corresponden a casos de atenuación pragmática. Al mismo tiempo, los resultados señalan que los discursos menos planificados prefieren una o dos estrategias prosódicas, habitualmente bajadas de intensidad, mientras que discursos más planificados, como el monólogo humorístico, usan todas las estrategias fónicas con igualdad.
\end{abstract}

Palabras clave: atenuación prosódica, planificación discursiva, géneros discursivos

\section{Introducción}

El objetivo principal de este artículo es estudiar la frecuencia y la tipología de la atenuación fónica en tres géneros discursivos distintos: la conversación coloquial, la entrevista televisiva y el monólogo humorístico. Para ello, se han recogido muestras orales de cada género con la finalidad de observar en qué casos, con respecto a una media contextual del hablante, acontece una disminución fónica significativa en el rango tonal, la intensidad y la velocidad de habla. Posteriormente, estos casos se han etiquetado o no como atenuación pragmática. 
Así, es un hecho comúnmente aceptado que la atenuación sirve para disminuir la fuerza ilocutiva de un acto de habla (Briz and Albelda 2013; Albelda, Briz, Cestero, Kotwica y Villalba 2014). Esto sucede cuando ese acto de habla puede ocasionar un daño a la imagen del oyente.

Los mecanismos lingüísticos para atenuar un bloque de habla son variados. Habitualmente, la bibliografía más reciente ha hecho referencia, sobre todo, a elementos léxicos o gramaticales, verbales en definitiva, sin tener en excesiva consideración el papel de la prosodia (Briz and Albelda 2013); aun así, algunos autores sí han señalado "the very important prosodic and kinesic means of mitigation, such as changes in pitch prominence, rhythm, speech rate, as well as eyecontact, gaze, gaze aversion, smile, particular postures, etc." (Caffi 1999: 890).

Si muchos de los estudios sobre atenuación no han acudido al análisis de la prosodia, es por un problema de identificación. Mientras que los elementos verbales pueden consultarse en un amplio repertorio de textos escritos o transcritos, no puede decirse lo mismo de los valores fónicos. Entre los diversos motivos de que esto sea así, pueden destacarse algunos, como el hecho de que los datos fónicos no suelen quedar reflejados correctamente en los corpus (CouperKuhlen and Selting 1996) o que, en ocasiones, el investigador pueda no manejar adecuadamente la diferencia entre lo fónico y lo paralingüístico (Wharton 2012).

Así mismo, tampoco es nada sencillo delimitar lo fónico desde un punto de vista puramente acústico. Por ejemplo, no existe un claro consenso en cuanto a la delimitación de unidades fónicas de análisis, si bien el mayor grueso de investigaciones (Hirschberg 2008; Prieto y Rigau 2011) sobre el discurso oral toman el grupo entonativo o frase entonacional (en términos de modelo TOBI) como punto de partida. Esto es, aquel segmento de habla que suele encontrarse delimitado por dos pausas o por dos inflexiones notables de la frecuencia fundamental (Quilis 1981; Szczepek Reed y Raymond 2013)

El mayor problema que acomete el investigador de lo oral es enfrentarse a aquello que, más allá de la fisonomía verbal, pueda acarrear significados concretos. En otras palabras, estructuras léxicas completamente neutras pueden revestirse de valores pragmáticos añadidos, como la atenuación, mediante la influencia de una variación prosódica en algún aspecto concreto, como la disminución de la Fo o el uso de grupos de entonación más breves (Hirschberg 2008; Wharton 2012). Tómese, como muestra, el Ejemplo (1) a continuación: 
(1) Ejemplo extraído de una conversación espontánea
A. ¡anda que tú tam(b)ién!
C. yo también noo ¡ella!
C. ella
A. tener unas llaves en casa y no decirlo
C. está cansada de revolicarlo to(do) y no sabía que ahí habían llaves
A. yy quien va y
A. $((\mathrm{cu}))$ yy
B. a veer
C. baaa

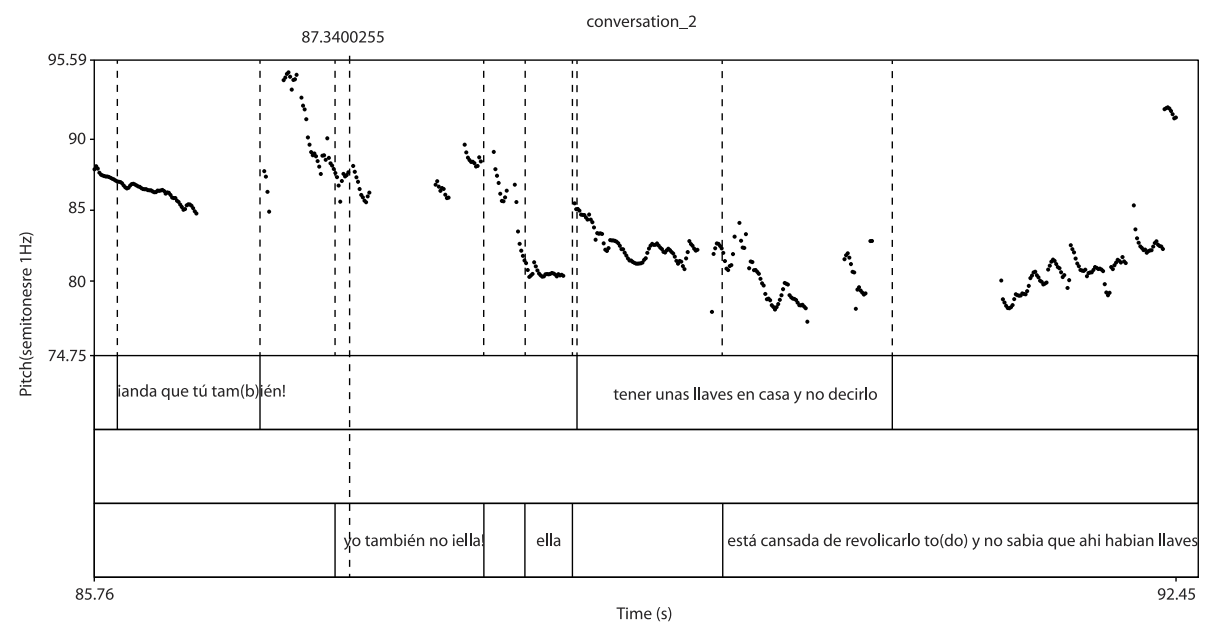

Figura 1. Curva melódica de la secuencia tener unas llaves en casa y no decirlo pronunciado en una Fo e intensidad significativamente inferiores a sus valores contiguos

En el ejemplo anterior, no es sencillo identificar la intervención de la hablante A tener unas llaves en casa y no decirlo como secuencia de atenuación si no se presta atención a su prosodia. Solo desde un punto de vista prosódico, esa intervención presenta una Fo de $115 \mathrm{~Hz}$, valor inferior a la media de la hablante A $(167.1 \mathrm{~Hz}$, con desviación estándar de $41.4 \mathrm{~Hz})$. Como ejemplo de atenuación no se contempla solo el descenso de la Fo o de la intensidad, sino que también se observa una protección de la imagen del hablante $\mathrm{C}$, que podría verse afectada por el comentario de A. De hecho, los valores de Fo e intensidad de la hablante A no solo son más bajos que sus registros generales, sino que presentan un descenso consecutivo a la secuencia de C yo también no jella!/ ella. En casos de desacuerdo o de contraste argumentativo, el hablante puede condicionar su propia elocución (Couper-Kuhlen y Selting 1996). 
Ahora bien, el descenso de los valores prosódicos no conlleva necesariamente un valor pragmático de atenuación, ya que puede a otros fenómenos lingüísticos, como los comentarios secundarios o incisos, las partes de una enumeración o las palabras finales de un turno de habla.

También cabe diferenciar entre atenuación y cortesía, ya que, "la atenuación (...) puede ser cortés (proteger la imagen ajena) o no serlo, y emplearse, entonces, para otros fines (como por ejemplo, proteger solo la imagen propia)" (Briz y Albelda 2010:239). Algunos autores han observado fenómenos prosódicos asociados con la expresión de la cortesía (Devís Herraiz 2012a, 2012b, 2014). Habitualmente, estos criterios suponen un aumento de la Fo (Ofuka et al. 200o) y de la velocidad de habla (Estellés 2013) en algunas zonas del grupo entonativo. No obstante, al menos en cuanto al incremento de los valores de la Fo, otros estudios no han encontrado una relación tan directa o determinante para la expresión de la cortesía (Brown y Prieto 2017).

Todo lo dicho anteriormente queda supeditado a la expectativa de los interlocutores en un contexto discursivo dado. Así, en general, podría pensarse que los géneros discursivos, en cuanto moldes o arquitecturas textuales, provocan el uso de elementos lingüísticos y estructuras prosódicas particulares; de este modo, los géneros discursivos se verían afectados por un mayor o menor control discursivo, condicionado, a su vez, por el grado de inmediatez discursiva (Briz and Albelda 2013; Albelda Marco et al. 2014).

\section{Recursos fónicos para la marcación de valores contextuales}

El habla se distribuye en el espacio contextual mediante trozos o piezas de emisión oral que, a su vez, pueden coincidir con estructuras sintácticas plenas; es lo que en la tradición del análisis conversacional se denomina turn constructional unit (Sacks, Schegloff y Jefferson 1974).

Por simple que esta estructura oral pueda resultar, en el habla espontánea no hay unanimidad en los factores fónicos que determinan su delimitación, ya que no solo la pausa puede convertirse en elemento demarcativo (Barnwell 2013). En general, la bibliografía más especializada establece que hay una combinación de variables que contribuyen a la delimitación de estas unidades fónicas: la velocidad de habla, la intensidad o volumen y el rango tonal (Couper-Kuhlen y Selting 1996; Selting 2010, 2000).

Al mismo tiempo, el cambio en estos factores no solo marca límites, sino que, tomados en consideración para toda la unidad, pueden aportar valores semánticos o pragmáticos específicos, como especifican algunos autores: 
Language researchers agree that intonation conveys various communicative functions that range from semantico-pragmatic functions such as speech act marking (assertion, question, etc.), information status (focus, given vs new information), belief status (epistemic position of the speaker with respect to the information exchange), politeness, affective, and emotional states, to indexical functions such as gender, age, and the sociolectal and dialectal status of the speaker.

(Prieto 2015:371)

Así, por ejemplo, en discurso oral la preocupación contenida de los hablantes ha sido vista como influida por "the combination of low and suppressed pitch range, low volume and relatively fast tempo plus a carrier which refers to an event as upsetting" (Couper-Kuhlen and Selting 1996: 26).

La cita anterior manifiesta una manera de estudiar la prosodia que atiende, sobre todo, a la configuración entonativa global del enunciado o acto de habla y no tanto a los patrones melódicos o acentuales que se observan en la curva de entonación. Se trata, por tanto, de una evolución del análisis de la conversación tradicional y se aparta, en cierta medida, de planteamientos más generalizados internacionalmente para el análisis prosódico, como pueda ser el modelo TOBI (Hirschberg 2008; Prieto and Rigau 2011) o el modelo INTSINT (Hirst, Di Cristo, and Espesser 2000).

En cuanto a los valores de rango tonal, velocidad o intensidad, la mayor parte de estudios hacen énfasis en géneros discursivos de alta preparación discursiva y donde, en gran parte de los casos, los hablantes leen directamente. Sería el caso, por ejemplo, de los estudios de las producciones fónicas de la radio o de la televisión (de la Mota and Rodero 2010; Rodero 2012). Otros estudios también han recogido esos valores fónicos para otros géneros discursivos, como la retransmisión deportiva, el discurso político o la conversación coloquial (Cabedo 2007).

Para la velocidad de habla, algunos autores sitúan la media general del español en 7.82 sílabas por segundo (Pellegrino, Coupé, and Egidio 2011); otros autores establecen distintos valores según el tipo de género discursivo en el que se insertan los correspondientes grupos entonativos. Por ejemplo, puede hablarse de 7.5 sílabas por segundo para la retransmisión deportiva o el discurso de un presentador televisivo, mientras que la entrevista y la conversación espontánea suelen tener una media de entre 6 y 6.5 sílabas por segundo (Deese 1984; Cabedo 2007).

En cuanto al rango tonal, se han hecho estudios particulares sobre otras lenguas distintas al español. Por ejemplo, en inglés y en francés los rangos tonales estriban entre 5 y 7 semitonos (Pépioto 2014). Para el español, otras investigaciones presentan una media general para todos los géneros discursivos de $10 \mathrm{a}$ 12 semitonos de media (Cabedo 2007). En cuanto a la percepción, a partir de 1.5 semitonos, el oído humano percibe cambio tonal (Martínez Celdrán y Fernández Planas 2007). 
Finalmente, sobre la intensidad o el volumen, puede hablarse, por poner un ejemplo, del umbral diferencial para el acento léxico, situado en $5 \mathrm{~dB}$ (Solé Sabater 1984). En estudios particulares sobre géneros discursivos (Cabedo 2007), se ha detectado una franja que oscila entre $77 \mathrm{~dB}$, dato establecido por géneros como la conversación o el discurso político, a unos $84 \mathrm{~dB}$, límite que ocupan géneros más ritualizados y expresivos como la retransmisión deportiva o el discurso de los presentadores de noticias televisivas.

\section{Diseño de la investigación}

En esta investigación se han recogido cinco muestras para cada género discursivo analizado. Las conversaciones proceden del corpus Val.Es.Co (Cabedo y Pons 2013); las entrevistas proceden de la televisión y han sido realizadas por el mismo entrevistador en dos cadenas españolas distintas (Cuatro y Antena 3); por último, los monólogos de humor han sido recogidos de un programa de televisión español llamado El club de la comedia.

En total, se han estudiado 21 hablantes. La distribución particularizada puede observar en la Tabla 1.

En la Tabla 1 aparecen 25 y no 21 hablantes, como se había comentado previamente. Esto sucede de esta manera porque las etiquetas I1_1, I2_1, I3_1, I4_1 y I5_1 corresponden al mismo sujeto, es decir, al entrevistador de las entrevistas televisivas. Se han mantenido en la tabla como hablantes distintos por diferenciar los valores de una entrevista a otra; en los análisis estadísticos este hablante se ha eliminado para no condicionar los resultados.

Sobre los minutos de grabación y palabras de este estudio, en total, se han recogido 74 minutos de grabaciones orales, compuestos por 3365 grupos entonativos y 18730 palabras. Estos datos se distribuyen de la siguiente manera:

- Conversación coloquial: 1686 grupos, duración total de 37 minutos y 9443 palabras.

- Entrevista: 761 grupos, duración total de 15 minutos y 3412 palabras;

- Monólogos humorísticos: 918 grupos, duración total de 22 minutos y 5875 palabras.

El estudio prosódico se ha realizado mediante el programa de análisis fonético PRAAT (Boersma and Weenink 2017); para poder recoger los valores acústicos, se ha hecho uso del script analyse tier (Hirst 2012) que, concretamente, permite el almacenamiento automático, para cada grupo entonativo, de los valores medios, máximo y mínimo de Fo e intensidad, así como del dato de la duración. 
Tabla 1. Distribución de valores por hablante (con información del género discursivo)

\begin{tabular}{|c|c|c|c|c|c|c|c|}
\hline \multirow[t]{2}{*}{ Hablantes } & \multirow{2}{*}{$\begin{array}{c}\text { Grupos entonativos } \\
\text { Frecuencia }\end{array}$} & \multicolumn{2}{|c|}{ Rango (St) } & \multicolumn{2}{|c|}{ Intensidad } & \multicolumn{2}{|c|}{ velocidad } \\
\hline & & Media & $\sigma$ & Media & $\sigma$ & Media & $\sigma$ \\
\hline C1_1 & 225.0 & 11.5 & 3.91 & 77.85 & 1.56 & 5.71 & 1.7 \\
\hline C1_2 & 228.0 & 10.5 & 4.26 & 76.31 & 3.23 & 6.74 & 2.3 \\
\hline C2_1 & 180.0 & 6.9 & 5.05 & 63.59 & 5.21 & 8.86 & 3.2 \\
\hline $\mathrm{C}_{2}{ }_{2}$ & 215.0 & $7 \cdot 3$ & 4.59 & 63.48 & 5.19 & 7.17 & 2.9 \\
\hline $\mathrm{C}_{3 \_1}$ & 80.0 & 7.9 & 6.36 & 62.70 & 5.65 & 5.97 & 2.9 \\
\hline $\mathrm{C}_{3 \_2}$ & 142.0 & 10.6 & 7.89 & 61.56 & 5.89 & 6.58 & 2.6 \\
\hline C4_1 & 183.0 & 12.1 & 5.00 & 74.37 & 6.70 & 6.30 & 3.1 \\
\hline $\mathrm{C} 4 \_2$ & 127.0 & 12.0 & 4.65 & 78.17 & 4.97 & 7.55 & 2.8 \\
\hline $\mathrm{C}_{5} \_1$ & 149.0 & $7 \cdot 5$ & $4 \cdot 37$ & 64.52 & 5.25 & 8.11 & 2.5 \\
\hline $\mathrm{C}_{5 \_2}$ & 90.0 & 10.0 & 5.53 & 64.76 & 5.14 & 6.88 & 2.8 \\
\hline H1_1 & 307.0 & 10.7 & 3.73 & 68.40 & 2.08 & 7.13 & 2.1 \\
\hline $\mathrm{H} 2 \_1$ & 228.0 & 10.1 & 3.38 & 71.54 & 1.69 & 8.51 & 2.4 \\
\hline $\mathrm{H}_{3}{ }_{-1}$ & 133.0 & 10.1 & 3.49 & 67.42 & 1.74 & 7.79 & 2.4 \\
\hline $\mathrm{H}_{4} \_1$ & 99.0 & 11.9 & 3.49 & 69.43 & 1.94 & 6.77 & 2.3 \\
\hline $\mathrm{H}_{5 \_1}$ & 146.0 & 9.9 & 4.14 & 70.11 & 2.86 & 7.02 & 2.6 \\
\hline I1_1 & 49.0 & 11.4 & 2.94 & 70.88 & 2.27 & 7.39 & 2.2 \\
\hline I1_2 & 97.0 & 9.1 & 3.69 & 67.26 & 3.95 & 7.24 & 2.4 \\
\hline I2_1 & 20.0 & 7.8 & 5.01 & 68.15 & 2.39 & 6.89 & 2.1 \\
\hline I2_2 & 87.0 & 9.7 & 3.54 & 66.90 & 2.94 & 5.84 & 2.1 \\
\hline I3_1 & 59.0 & 10.6 & 3.85 & $69 \cdot 36$ & 3.40 & 7.88 & 2.7 \\
\hline I3_2 & 88.0 & 10.1 & 4.53 & 69.93 & 3.24 & 6.03 & 3.2 \\
\hline I4_1 & 62.0 & 13.0 & 3.59 & 74.50 & 3.17 & 6.36 & 2.8 \\
\hline I4_2 & 149.0 & 9.9 & 3.77 & 73.17 & $3 \cdot 38$ & 6.80 & $3 \cdot 3$ \\
\hline I5_1 & 58.0 & 11.3 & 3.97 & 69.38 & 3.52 & 6.54 & 2.7 \\
\hline I5_2 & 90.0 & 9.0 & 3.94 & 69.21 & 3.87 & 5.16 & 1.9 \\
\hline
\end{tabular}

Posteriormente, en la base de datos se han realizado diferentes operaciones matemáticas para obtener otros valores, como la velocidad de habla (medida en sílabas por segundo) o el rango tonal (procedente de la resta entre la Fo máxima y la mínima). Este último ha sido incorporado en semitonos, después de aplicar la fórmula correspondiente de conversión de los $\mathrm{Hz}$ (Martínez Celdrán and Fernández Planas 2007), es decir, $12^{*} \log _{2}$ (fomax/fomin) para el rango tonal. 
El procedimiento de análisis ha sido el siguiente: los valores fónicos recogidos de cada grupo entonativo (velocidad de habla, rango tonal, intensidad media) han sido comparados con la media correspondiente del hablante; se ha considerado disminución fónica si la diferencia entre el valor del grupo y la media del hablante resultaba inferior a una desviación típica multiplicada por 1.65, valor que marca el límite para una diferencia estadísticamente significativa (Moore y McCabe 1999).

Cabe hacer la salvedad de que para la velocidad de habla, tal y como apuntan algunos autores (Ofuka et al. 200o; Estellés 2013), los valores de velocidad elocutiva pueden ser también significativamente superiores a la media; es por ello por lo que, en cuanto a esta variable, se ha tenido en consideración tanto la ralentización como el incremento en la velocidad de habla.

Tómese como ejemplo el registro 89 de la base de datos, del hablante C1_1, ¿pero qué dices?, con un rango tonal de 4,35 st. La media de ese hablante para el rango tonal es de 11.5 st y una desviación típica de 3.9 st. Así, dado que la diferencia es de $-7,15$ st, este valor es inferior a multiplicar 3.9 st por 1.65 (resultado $6.4 \mathrm{st}$ ). En definitiva, puede establecerse que, en cuanto al rango tonal, este grupo entonativo se aparta en gran medida de la media del hablante en el contexto interactivo registrado.

De este modo, solo se han tenido en cuenta los grupos entonativos que, en alguno de los tres aspectos fónicos analizados, se hayan distanciado sustancialmente de los valores medios del hablante. Como muestra del procedimiento, puede observarse la Figura 2; en ella, se observa la velocidad de habla de los 30 primeros grupos entonativos del hablante $\mathrm{C}_{1-1}$. La línea vertical, en forma de torre, recoge la desviación estándar del hablante (1.7 sílabas por segundo) en relación a la media ( 5.71 sílabas/segundo) y el eje de ordenadas proporciona el dato concreto de velocidad para cada grupo entonativo. Algunos de esos 30 valores quedan por encima de la desviación estándar, tanto por debajo $(3.4,3.7,4.3)$ como por arriba $(7.4,6.8,6.6,6.7,7.8,7.1)$.

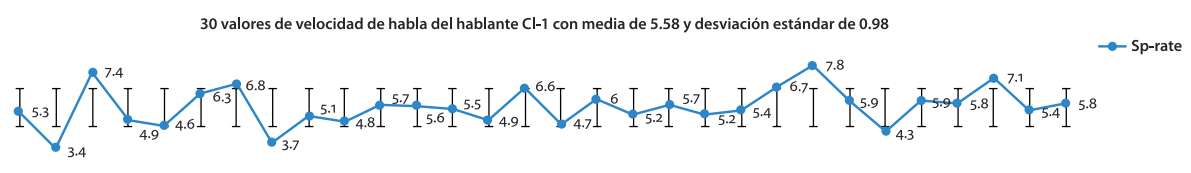

C1-1 C1-1 C1-1 C1-1 C1-1 C1-1 C1-1 C1-1 C1-1 C1-1 C1-1 C1-1 C1-1 C1-1 C1-1 C1-1 C1-1 C1-1 C1-1 C1-1 C1-1 C1-1 C1-1 C1-1 C1-1 C1-1 C1-1 C1-1 C1-1 C1-1

Figura 2. Velocidad de habla de los 30 primeros grupos entonativos del hablante $\mathrm{C}_{1-1}$, con media de 5.58 sílabas/segundo y desviación estándar de 0.98

Debe destacarse un aspecto metodológico particular. El hecho de que se haya tomado 1.65 desviaciones típicas en relación con la media del hablante no conlleva necesariamente que pueda atenuarse también, incluso solo fónicamente, usando 
un valor, por ejemplo, algo inferior. No obstante, y en aras de homogeneizar los datos tanto por hablante como por género, se ha decidido usar este y no otro criterio de discriminación.

Así, podría darse la circunstancia de que un grupo entonativo tuviera un rango tonal 3 semitonos por debajo de la media (dato que es perceptivo por el oído humano), pero si la desviación típica del hablante es 2, no se ha tenido en cuenta en los resultados finales.

Con ello, quiere insistirse en el hecho de que, a nivel perceptivo, los casos de atenuación fónica podrían ser mayores si el punto de comparación no fuera la media del propio hablante, sino la del género correspondiente que se haya establecido previamente por la bibliografía; por ejemplo, 6-7 sílabas por segundo para la conversación (Deese 1984).

Finalmente, cada uno de los datos catalogados como significativamente inferiores a la media del hablante se ha etiquetado como "atenuación o no atenuación". Por tanto, en todos los géneros hay casos de grupos entonativos que tienen un valor muy bajo de intensidad o muy alto de velocidad de habla y que, sin embargo, no constituyen atenuación pragmática. Por ejemplo, los titubeos, reinicios, las parcelaciones del discurso por no encontrar la palabra adecuada, etc., pueden conllevar un descenso de los valores fónicos, pero no por ello pueden considerarse atenuación. Dicho de otra manera: el descenso acentuado de los valores fónicos puede o no conllevar atenuación y viceversa.ç

\section{Selección de casos por factor fónico y atenuación}

En primer lugar, los grupos entonativos se han seleccionado según su desviación respecto a la media del hablante para cada factor fónico investigado. Posteriormente, cada uno de estos grupos ha sido analizado contextualmente para observar básicamente dos aspectos: la minimización de la fuerza ilocutiva del grupo entonativo y, también, la repercusión que esto pudiera tener en la imagen, bien del hablante, bien del interlocutor o de otra persona no presente en la conversación.

Se siguen, de este modo, los criterios básicos de identificación de la atenuación pragmática (Albelda Marco et al. 2014; Briz y Albelda 2013) y se añaden otros criterios como, por ejemplo, cuál sería la repercusión de omitir o no la posible marca de atenuación (fónica en este caso) y, por tanto, la presencia o ausencia de una posterior atenuación (Villalba 2016).

Para ilustrar el proceso de catalogación de la base de datos de este estudio, se exponen a continuación una serie de ejemplos. Se trata de casos que presentan algún rasgo prosódico minimizado (o también aumentado, en el caso de la velocidad de habla) y que han sido evaluados, finalmente, como atenuación. 
(2) Ejemplo extraído de una entrevista televisiva (hablante I1_2)

B. le debo mii (o.69)

B. mi falta de autoestima en algunas ocasiones (RISAS) (0.32)

B. le deboo (o.39)

B. mis- mis frustraciones

B. le deboo mi afán competitivo y luchador yy (o.19)

B. y tenaz

B. y perseverancia

B. se la debo a él

(2.87 sílabas por segundo; media del hablante, 7.2, desviación típica: 2.4 )

En el Ejemplo (2), el entrevistado está relatando un momento pasado en el que, previamente a la adicción de sustancias dopantes, su primer entrenador de waterpolo fue alguien importante para él. La velocidad de habla de la secuencia mismis frustraciones tiene una velocidad de habla lenta porque el hablante busca una palabra que no encuentra (de ahí el reinicio). También se ralentiza el habla porque el grupo entonativo provoca una afrenta a la imagen del entrenador, al que se culpa inicialmente de esas frustraciones; de hecho, el propio hablante corrige inmediatamente su posible amenaza a la imagen del otro. Esta restitución se realiza con una secuencia de cuatro grupos entonativos consecutivos que alaban, precisamente, la figura del mismo entrenador.

Por otro lado, los casos de velocidad de habla rápida son también interesantes. El Ejemplo (3) es muestra de ello:

(3) Ejemplo extraído de una entrevista (hablante I3_1)

A. para ser un buen chef

A. ¿hace falta estar gordo?

(o.93)

B. no

$(0.42)$

B. no necesariamente creo que cada uno tienee suu manera dee (0.47)

B. de asimilar las cosas eem de hecho hay ahora unaa (0.37)

B. muy buena colección de cocineros

(o.38)

B. quee

(0.29) 
B. que aparte de ser grandísimos cocineros

(o.10)

B. pues soon

(o.14)

B. muy buenos deportistas

(0.05)(17.6 sílabas por segundo; media del hablante, 7.88; desviación típica: 2.7)

En el Ejemplo (3) el entrevistador, después de algunos preámbulos de distensión discursiva con el entrevistado, comienza su entrevista con la pregunta para ser un buen chef / ¿hace falta estar gordo? Esta pregunta supone un inicio marcadamente descortés, sin embargo, el entrevistador, que sabe que está atentando contra la imagen del entrevistado, acelera la velocidad elocutiva. Por tanto, se trata de un caso de atenuación pragmática que intenta minimizar, en primer lugar, el hecho de buscar una respuesta mediante una pregunta directa y, en segundo lugar, el de incomodar al entrevistado mediante la crítica al aspecto físico de este.

A continuación, el Ejemplo (4) procede de una reducción del rango tonal del hablante:

(4) Ejemplo extraído de una conversación (hablante C5_2)

A. total que mi padre estaa

(o.28)

A. cagao preocupao y de todo

(o.69)

A. y mi madre también está preocupada

(1.55)

B. dios le conceda una muerte repentina de infarto

(o.40)

B. si no nos va a dar este hombre

(1.14)

A. sí será que [te enteras] tú mucho

B. $[(())]$

(o.52)

A. de lo que te da

(0.35)

B. no pero da igual solo verle la cara yaa

(2.58)

C. ¿quéé? $<$ ta $>$

(o.79)

A. hay-hay un trozo de sobra [¿queréis poneros?] <ta $>$

B. [se caga el abuelo]

(Rango tonal: 1.82 st; media del hablante, 6.9 st; desviación típica: 2.8 st) 
En el ejemplo anterior, A y B están hablando sobre la situación del padre de A, ya mayor y con problemas de salud. B ha emitido previamente una secuencia despectiva e irrespetuosa dios le conceda una muerte repentina de infarto, a la que sigue una queja por la posibilidad de tener que cuidar al padre de A en el futuro. A responde con un sí será que [te enteras] tú mucho; en la siguiente intervención B intenta reparar su propia imagen con un no pero da igual y utiliza una estrategia de atenuación fónica, reduciendo el espacio del rango tonal sustancialmente.

Finalmente, el Ejemplo (5) muestra un caso de reducción significativa de la intensidad:

(5) Ejemplo extraído de un monólogo de humor (Hablante H1_1)

A. a ver yo tengo un déficit de cariño

A. pero es algo histórico mío

A. tampoco quiero aburrir (0.56)

A. pero en mi casa noo

A. a mí no me quisieron

A. en mi familia no me querían (1.22)

P. (RISAS)

A. esto no es un chiste cabrones (2.03)

P. (RISAS)

A. no es que haay- no perdón es que hay unos que van desacompasa(d)os (1.11)(Intensidad media: $63 \mathrm{~dB}$; media del hablante, $68.4 \mathrm{~dB}$; desviación típica: $2.1 \mathrm{~dB}$ )

En el ejemplo anterior, el humorista comienza su monólogo atacando su propia imagen y manifestando una baja autoestima fingida (en mi familia no me querían). Ante las risas del público, que es el efecto perlocutivo perseguido por el humorista, se expone, de nuevo, una fingida manifestación de ofensa personal. Esto se realiza con una secuencia aparentemente descortés: esto no es un chiste cabrones. El descenso de intensidad en el grupo entonativo provoca, de nuevo, el efecto perseguido: la risa del espectador.

\section{Análisis y resultados. Disminución de valores prosódicos y atenuación}

Los resultados de esta investigación incluyen los grupos entonativos de la base de datos cuyos valores han sido significativamente distintos de la media del hablante; en concreto, todos los grupos que presenten valores con una distancia inferior de 1.65 desviaciones típicas en relación a la media del hablante. 
Para observar la relación entre género, disminución fónica y atenuación, puede consultarse la Tabla 2:

Tabla 2. Distribución por género de la atenuación fónica, la fónico-pragmática y la ausencia de atenuación

\begin{tabular}{|c|c|c|c|c|c|c|c|c|}
\hline \multirow[b]{3}{*}{ Atenuación } & \multicolumn{6}{|c|}{ Géneros } & \multirow{3}{*}{ Total } & \multirow{3}{*}{ Total\% } \\
\hline & \multicolumn{2}{|c|}{ Conversación } & \multicolumn{2}{|c|}{ entrevista } & \multicolumn{2}{|c|}{ humor } & & \\
\hline & Frec. & $\%$ & Frec. & $\%$ & Frec. & $\%$ & & \\
\hline Solo fónica & 157 & $9.70 \%$ & 60 & $7.91 \%$ & 81 & $8.87 \%$ & 298 & $9.05 \%$ \\
\hline $\begin{array}{l}\text { Fónica y } \\
\text { pragmática }\end{array}$ & 99 & $6.11 \%$ & 54 & $7.11 \%$ & 84 & $9.20 \%$ & 237 & $7.20 \%$ \\
\hline $\begin{array}{l}\text { No atenuación } \\
\text { fónica }\end{array}$ & 1363 & $84.19 \%$ & 645 & $84.98 \%$ & 748 & $81.93 \%$ & 2756 & $83.74 \%$ \\
\hline Total general & 1619 & $100 \%$ & 759 & $100 \%$ & 913 & $100 \%$ & 3291 & $100 \%$ \\
\hline
\end{tabular}

En la Tabla 2 se establece la distribución de la atenuación en grupos entonativos con variables fónicas que presentan valores por debajo de la media del hablante. Debe especificarse que, para la atenuación pragmática, cada uno de los datos indica que, por lo menos, hay un mínimo de un factor fónico afectado. Así, por ejemplo, si un grupo entonativo se cataloga como atenuado y presenta, además, ralentización de la velocidad elocutiva y descenso del rango tonal, se ha considerado como un solo caso, ya que, como se señalaba, es solo uno el grupo entonativo atenuado.

No se ha encontrado una relación significativa de dependencia entre el género y el tipo de atenuación empleada $\left(X_{2}=5.08 ; d f=2 ; p=0.08\right)$; así pues, en cada género, la distribución de atenuación fónica y de descenso de los valores fónicos sin atenuación tiene un comportamiento similar. Al mismo tiempo, si solo se analiza la variante de atenuación, tampoco se observan distribuciones significativamente distintas $\left(X_{2}=3.99 d f=2, p=0.126\right)$.

Si se sigue atendiendo a los datos de la Tabla 2, puede observarse que los tres géneros manifiestan un porcentaje similar de habla no afectada por una especial modificación de de la velocidad, el rango tonal o la intensidad. Este porcentaje estriba en un 8o-85\%; como curiosidad, son dos géneros de menor y mayor preparación discursiva, respectivamente, los que presentan mayores alteraciones entonativas. Estos géneros son la conversación y el monólogo humorístico.

Así pues, puede deducirse que los hablantes, en la elaboración de su discurso, una vez han asumido los presupuestos tanto del género como del contexto, mantienen generalmente los valores fónicos de su elocución; dejan por tanto las alteraciones prosódicas para situaciones discursivas específicas. Se recuerda aquí 
lo ya expuesto por otros autores, en tanto en cuanto lo contextualmente marcado se expresa de una manera formalmente marcada (Levinson 1983).

La Tabla 2 puede subdividirse todavía más en los valores que aparecen en la Tabla 3. En general, se trata de los mismos valores, pero sin tener en cuenta el $80-85 \%$ de casos en los que la entonación no aparecía modificada.

Tabla 3. Distribución de los mecanismos vinculados con la modificación significativa de la intensidad, el rango o la velocidad de habla en casos de atenuación pragmática

\begin{tabular}{|c|c|c|c|c|c|c|c|c|c|c|}
\hline \multirow[b]{2}{*}{ rango } & \multirow[b]{2}{*}{ Veloc. } & \multirow[b]{2}{*}{ Inten. } & \multicolumn{2}{|c|}{ Conversación } & \multicolumn{2}{|c|}{ entrevista } & \multicolumn{2}{|c|}{ humor } & \multirow[b]{2}{*}{ Total } & \multirow[b]{2}{*}{ Total\% } \\
\hline & & & Frec. & $\%$ & Frec. & $\%$ & Frec. & $\%$ & & \\
\hline o & 0 & A & 36.0 & $36 \%$ & 14.0 & $26 \%$ & 24.0 & $29 \%$ & 74.0 & $31 \%$ \\
\hline o & A & o & 14.0 & $14 \%$ & 5.0 & $9 \%$ & 9.0 & $11 \%$ & 28.0 & $12 \%$ \\
\hline o & A & A & 1.0 & $1 \%$ & & O\% & 1.0 & $1 \%$ & 2.0 & $1 \%$ \\
\hline o & A & I & & $0 \%$ & 1.0 & $2 \%$ & & o\% & 1.0 & o\% \\
\hline o & I & 0 & 27.0 & $27 \%$ & 15.0 & $28 \%$ & 17.0 & $20 \%$ & 59.0 & $25 \%$ \\
\hline o & I & A & 2.0 & $2 \%$ & & o\% & & o\% & 2.0 & $1 \%$ \\
\hline o & I & I & 2.0 & $2 \%$ & 1.0 & $2 \%$ & 1.0 & $1 \%$ & 4.0 & $2 \%$ \\
\hline A & o & 0 & 8.0 & $8 \%$ & 9.0 & $17 \%$ & 17.0 & $20 \%$ & 34.0 & $14 \%$ \\
\hline A & o & A & 6.0 & $6 \%$ & 4.0 & $7 \%$ & 5.0 & $6 \%$ & 15.0 & $6 \%$ \\
\hline A & o & I & & o\% & & O\% & 1.0 & $1 \%$ & 1.0 & O\% \\
\hline A & A & 0 & & O\% & & O\% & 4.0 & $5 \%$ & 4.0 & $2 \%$ \\
\hline A & I & o & 1.0 & $1 \%$ & 1.0 & $2 \%$ & & O\% & 2.0 & $1 \%$ \\
\hline A & I & A & 2.0 & $2 \%$ & & O\% & & O\% & 2.0 & $1 \%$ \\
\hline I & 0 & A & & O\% & 3.0 & $6 \%$ & 3.0 & $4 \%$ & 6.0 & $3 \%$ \\
\hline I & A & 0 & & O\% & 1.0 & $2 \%$ & & O\% & 1.0 & O\% \\
\hline I & A & A & & $0 \%$ & & O\% & 1.0 & $1 \%$ & 1.0 & O\% \\
\hline I & I & o & & o\% & & O\% & 1.0 & $1 \%$ & 1.0 & O\% \\
\hline $\begin{array}{l}\text { Total } \\
\text { general }\end{array}$ & & & 99.0 & $100 \%$ & 54.0 & $100 \%$ & 84.0 & $100 \%$ & 237.0 & $100 \%$ \\
\hline
\end{tabular}

En la Tabla 3, por tanto, aparecen en combinación complementaria tres posibilidades: los casos en los que no hay un aumento o descenso de la cadena fónica, catalogados como o, con los que presentan un incremento significativo, catalogados con la I de intensificación, y los que incluyen disminución fónica, catalogados con la A de atenuación.

Cabe recordar que en la Tabla 3 solo aparecen los casos en los que se ha detectado una disminución del rango tonal o de la intensidad; en el caso de la velocidad 
de habla, los datos pueden ser superiores o inferiores a la media. Así mismo, en las combinaciones que se observan, también aparecen casos en los que, para el rango tonal o la intensidad, los valores puedan estar intensificados. Así, la combinación IoA se ha incluido en la Tabla porque tiene un valor de disminución para la intensidad, pero, por el contrario, tiene al mismo tiempo un incremento de rango tonal. Estos casos han resultado ser residuales por lo general.

En cuanto a los mecanismos de atenuación fónica empleados, los hablantes recogidos en la base de datos utilizan prioritariamente los cuatro mecanismos analizados de forma aislada. Del total de grupos entonativos con disminución prosódica, un $12 \%$ utiliza una velocidad de habla baja; un $25 \%$, velocidad de habla alta; el $31 \%$ hace uso de la intensidad baja; y, finalmente, el 14\% presenta reducción de rango tonal.

Por todo lo visto en el corpus analizado, no puede hablarse de géneros discursivos más atenuados fónicamente que otros. De hecho, los tres presentan un comportamiento muy similar. Únicamente parece haber algunas especializaciones de los procedimientos prosódicos utilizados. Así, mientras la entrevista y la conversación hacen uso, básicamente, de una menor velocidad de habla y de una menor intensidad, el monólogo de humor, en tanto en cuanto discurso más elaborado, utiliza los anteriores y, con mayor uso, el descenso en el rango tonal.

Por lo tanto, aunque no resulta todo lo sistemático que pudiera esperarse (cabe tener en cuenta que se trata de un estudio de corpus de habla real), los datos apuntan a una mayor especialización prosódica según el grado de planificación del discurso: géneros discursivos más planificados utilizan mayores recursos fónicos que aquellos, como la conversación y la entrevista, en los que predomina un mayor grado de espontaneidad. Todo ello puede observarse en la Tabla 4:

Tabla 4. Síntesis de los factores fónicos utilizados según género

\begin{tabular}{lccc}
\hline conversación & entrevista & humor & \\
\hline $0.93 \%$ & $0.92 \%$ & $1.64 \%$ & velocidad(A) \\
$2.10 \%$ & $2.24 \%$ & $1.64 \%$ & velocidad(I) \\
$1.05 \%$ & $1.84 \%$ & $2.96 \%$ & Rango \\
$2.90 \%$ & $2.77 \%$ & $3.72 \%$ & Intensidad \\
\hline
\end{tabular}

La Tabla 4 establece el porcentaje de grupos entonativos por género que se ven afectados por una disminución estadísticamente significativa en sus valores. En el caso de la velocidad de habla, se ha analizado tanto el incremento como el descenso de la velocidad, ya que ambos pueden conllevar estrategias de atenuación.

Así, por ejemplo, el monólogo de humor presenta valores porcentuales elevados para todas las variables analizada, aproximadamente de un $2-4 \%$. En 
el caso de la entrevista, también hay valores altos en todas las variables menos en velocidad de habla baja. Finalmente, en la conversación espontánea, son solo el aumento de la velocidad y de la intensidad los criterios fónicos que alcanzan porcentajes elevados de un $2-3 \%$.

De esta manera, parece obvio que los discursos más planificados utilizan un repertorio ligeramente mayor de mecanismos fónicos para expresar atenuación que los discursos más espontáneos. No debe confundirse, en todo caso, la tipología de los recursos utilizados con la frecuencia global, ya que todos los géneros analizados en este estudio manifiestan la misma proporción de grupos entonativos atenuados fónicamente.

\section{Conclusiones}

Este estudio ha analizado recursos prosódicos (velocidad de habla, rango tonal e intensidad) que participan en la minimización perceptiva de algunas unidades fónicas en el discurso hablado. Así mismo, ese descenso de los valores prosódicos se ha puesto en relación con la atenuación pragmática implicada, entendida como la minimización de la fuerza ilocutiva de los actos de habla (Briz y Albelda 2013).

Un total de 3366 grupos entonativos se han extraído de tres géneros discursivos distintos: la conversación coloquial, la entrevista televisiva y el monólogo humorístico. Estos géneros han sido seleccionados por presentar un distinto grado de planificación discursiva.

A diferencia de otros estudios en los que los valores particulares de cada individuo se comparan con los de una media previamente establecida (Cabedo 2007; de la Mota y Rodero 2010; Rodero 2012; Deese 1984), en este estudio se han tomado en cuenta aquellos grupos entonativos que se diferenciaban no de una media general, sino de la media particular de cada valor con respecto a la media contextual del hablante. Además, en aras de buscar una representatividad de los datos, solo se han tenido en cuenta aquellos datos que se diferenciaban significativamente de la media, ya que se apartaban en 1.65 desviaciones típicas como mínimo (Moore y McCabe 1999).

Los resultados generales no indican que haya géneros que atenúen más que otros fónicamente, el nivel de disminución prosódica coincide en cada género en un $15-20 \%$. De este porcentaje, la mitad corresponde, además, a atenuación pragmática.

Lo que sí se ha observado, y parece proceder del grado de planificación discursiva, es el hecho de que los géneros discursivos más elaborados reparten proporcionalmente el tipo de procedimiento de disminución prosódica que emplean. Al contrario, los menos planificados presentan una mayor polarización, 
sobre todo centrada en el descenso de la intensidad y en el aumento de la velocidad de habla.

En futuros estudios debe indagarse todavía más en la correspondencia entre la minimización fónica y el tipo de procedimiento verbal empleado o, también, en explorar no solo las unidades fónicas que se distancien significativamente de la media del hablante, sino aquellas que presentan desviaciones menores y que, perceptivamente, se consideran atenuación fónica. Quizá, incluso, podrían definirse márgenes acústicos que diferenciaran la disminución fónica sin vinculación pragmática de aquella que se utiliza para la expresión de la atenuación o, incluso, de otros valores pragmáticos.

\section{Reconocimientos}

Este trabajo ha sido desarrollado gracias a la financiación recibida en el proyecto "La atenuación pragmática en su variación genérica: géneros discursivos escritos y orales en el español de España y América" (MINECO, FFI2016-75249-P).

\section{Referencias}

Albelda Marco, Marta, Antonio Briz Gómez, Ana María Cestero Mancera, Dorota Kotwica y Cristina Villalba Ibáñez. 2014. "Ficha metodológica para el análisis pragmático de la atenuación en corpus discursivos del Español (Es.por.atenuación).” Oralia: Análisis Del Discurso Oral 17: 7-62.

Barnwell, Brendan. 2013. "Perception of Prosodic Boundaries by Untrained Listeners." In Units of Talk - Units of Action, ed. por Beatrice Szczepek Reed y Geoffrey Raymond, 125-66. John Benjamins Publishing Company. https://doi.org/10.1075/slsi.25.05bar.

Boersma, Paul y David Weenink. 2017. "Praat: Doing Phonetics by Computer." http://www.praat.org/.

Briz, Antonio y Marta Albelda. 2010. “Aspectos pragmáticos. Cortesía y atenuantes verbales en las dos orillas a través de muestras orales." En La lengua española en América: Normas y usos actuales, ed. por Milagros Aleza y José María Enguita, 237-60. Valencia: Servei de Publicacions de la Universitat de València.

Briz, Antonio y Marta Albelda. 2013. "Una propuesta teórica y metodológica para el análisis de la atenuación lingüística en español y portugués. La base de un proyecto en vomún (ES.POR. ATENUACIÓN)." Onomazein 28 (2): 288-319. https://doi.org/10.7764/onomazein.28.21.

Brown, Lucien y Pilar Prieto. 2017. “(Im)politeness: Prosody and Gesture." En The Palgrave Handbook of Linguistic (Im)politeness, 357-79. London: Palgrave Macmillan UK. https://doi.org/10.1057/978-1-137-37508-7_14.

Cabedo, Adrián. 2007. "Marcas prosódicas del registro coloquial en la conversación." Cauce 30: $41-56$. 
Cabedo, Adrián y Salvador Pons. 2013. "Corpus Val.Es.Co 2.o." www.valesco.es.

Caffi, Claudia. 1999. "On Mitigation." Journal of Pragmatics 31 (7): 881-909. https://doi.org/10.1016/S0378-2166(98)00098-8.

Couper-Kuhlen, Elizabeth y Margret Selting. 1996. “Towards an Interactional Perspective on Prosody and a Prosodic Perspective on Interaction." En Prosody in Conversation 11-56. https://doi.org/10.1017/CBO9780511597862.003.

Deese, James. 1984. Thought into Speech: The Psychology of a Language. Englewood Cliffs, NJ: Prentice-Hall.

Devís Herraiz, Empar. 2012a. “Cortesía atenuadora en el español coloquial.” Cauce 34-35: 117-38.

Devís Herraiz, Empar. 2012b. "Percepción de la cortesía atenuadora en el español coloquial." Oralia: Análisis Del Discurso Oral 15: 125-45.

Devís Herraiz, Empar. 2014. “The Intonation of Mitigating Politeness in Catalan." Journal of Politeness Research 10 (1): 127-49. https://doi.org/10.1515/pr-2014-0006

Estellés, María. 2013. "La expresión fónica de la cortesía en tribunales académicos." Oralia: Análisis Del Discurso Oral 16: 81-110. Editorial ARCO Libros e ILSE.

Hirschberg, Julia. 2008. "Pragmatics and Intonation." En The Handbook of Pragmatics, ed. por Laurence Horn y Gregory Ward, 515-37. Oxford: Blackwell.

Hirst, Daniel. 2012. “Analyse Tier.” https://groups.yahoo.com/group/praat-users.

Hirst, Daniel, Albert Di Cristo y Robert Espesser. 200o. "Levels of Representation and Levels of Analysis for the Description of Intonation Systems." En Prosody: Theory and Experiment ed. por Merle Horne, 51-87. Netherlands: Springer. https://doi.org/10.1007/978-94-015-9413-4_4

la Mota, Carme de y Emma Rodero. 2010. "La demarcación entonativa y el énfasis en la locución de los rditores de noletinesinformativos radiofónicos - Dialnet." En XXXIX Simposio Internacional de La Sociedad Española de Lingüistica (SEL) ed. por SEL, 1-69. Santiago de Compostela: SEL. https://dialnet.unirioja.es/servlet/articulo? codigo=5412029.

Levinson, S. C. 1983. Pragmatics. Cambridge: Cambridge University Press.

Martínez Celdrán, Eugenio y Ana María Fernández Planas. 2007. Análisis espectrográfico de los sonidos del aabla. Barcelona: Ariel.

Moore, David S. y George P. McCabe. 1999. Introduction to the Practice of Statistics. W. H. Freeman.

Ofuka, Etsuko, J. Denis McKeown, Mitch G. Waterman y Peter J. Roach. 20oo. "Prosodic Cues for Rated Politeness in Japanese Speech." Speech Communication 32 (3): 199-217. https://doi.org/10.1016/S0167-6393(00)00009-1.

Pellegrino, François, Christophe Coupé y Marsico Egidio. 2011. "A Cross-Language Perspective on Speech Information Rate." Language 87 (3). Linguistic Society of America: 539-58. http://www.jstor.org/stable/23011654.

Pépioto, Erwaa. 2014. "Male and Female Speech: A Study of Mean fo, fo Range, Phonation Type and Speech Rate in Parisian French and American English Speakers." Speech Prosody 7. https://halshs.archives-ouvertes.fr/halshs-0o999332.

Prieto, Pilar. 2015. "Intonational Meaning." Wiley Interdisciplinary Reviews: Cognitive Science 6 (4): 371-81. https://doi.org/10.1002/wcs.1352.

Prieto, Pilar y Gemma Rigau. 2011. "Prosody and Pragmatics." En The Pragmatics of Catalan ed. por Lluís Payrató y Josep Maria Cots, 17-48. The Hague: Mouton de Gruyter. https://doi.org/10.1515/9783110238693.17 
Quilis, Antonio. 1981. Fonética Acústica de La Lengua Española. Madrid: Biblioteca Románica Hispánica.

Rodero, Emma. 2012. "A Comparative Analysis of Speech Rate and Perception in Radio Bulletins.” Text \& Talk. https://doi.org/10.1515/text-2012-0019.

Sacks, Harvey, Emanuel A. Schegloff y Gail Jefferson. 1974. "A Simplest Systematics for the Organization of Turn-Taking for Conversation." Language 50 (4): 696. https://doi.org/10.2307/412243.

Selting, Margret. 2000. "The Construction of Units in Conversational Talk." Language in Society 29: 477-517. https://doi.org/10.1017/S0047404500004012.

Selting, Margret. 2010. "Prosody in Interaction: State of the Art." In Prosody in Interaction, 3-40. https://doi.org/10.1075/sidag.23.05sel

Solé Sabater, Maria Josep. 1984. "Experimentos Sobre la percepción del acento." Estudios de Fonética Experimental 1. Universitat de Barcelona: 131-242. http://www.raco.cat/index.php/EFE/article/view/144192/256820.

Szczepek Reed, Beatrice y Geoffrey Raymond. 2013. Units of Talk-Units of Action. Amsterdam: John Benjamins Publishing Company. https://doi.org/10.1075/slsi.25.

Villalba, Cristina. 2016. "Actividades de imagen, atenuación e impersonalidad en los juicios orales." Universitat de València.

http://roderic.uv.es/bitstream/handle/10550/54010/Cristina

Villalbatesisdoctoral.pdf? sequence $=1$ \&isAllowed $=\mathrm{y}$.

Wharton, Tim. 2012. "Pragmatics and Prosody." En The Cambridge Handbook of Pragmatics ed. por Keith Allan y Kasia M. Jaszczolt, 567-84. Cambridge: Cambridge University Press. https://doi.org/10.1017/CBO9781139022453.031.

\title{
Address for correspondence
}

\author{
Adrian Cabedo \\ Grupos Val.Es.Cu \\ Universitat de València \\ Avda. Blasco Ibáñez 32 \\ 46010 Valencia \\ España
}

Adrian.cabedo@uv.es 